\title{
Characterization of the microbiomic association between tonsil and saliva isolated from Korean children subjected to tonsillectomy using 16S rRNA sequencing: a pilot study
}

\section{Da Hyeon Choi}

Chungbuk National University

Ji Won Park

Chungbuk National University

Ju Kwang Choi

Chungbuk National University

Kyeong Eun Lee

Chungbuk National University

Won Hee Lee

Institute of MD Healthcare Inc

Jinho Yang

Institute of MD healthcare Inc

Ju Yeon Lee

Nano Intelligent Biomedical Engineering Corporation (NIBEC)

Yoon Jeong Park

Seoul National University

Chan Oh

Chungnam National University School of Medicine

Ho-Ryun Won

Chungnam National University School of Medicine

Bon Seok Koo

Chungnam National University School of Medicine Jae Won Chang

Chungnam National University School of Medicine Yoon Shin Park ( $\nabla$ pys@cbnu.ac.kr)

Chungbuk National University https://orcid.org/0000-0002-5373-9218

Research 
Keywords: Microbiome, Saliva, Tonsil, Tonsillectomy

Posted Date: April 8th, 2020

DOI: https://doi.org/10.21203/rs.3.rs-20841/v1

License: (c) (1) This work is licensed under a Creative Commons Attribution 4.0 International License. Read Full License 


\section{Abstract}

\section{Background}

Oral microbes have the capacity to spread throughout the gastrointestinal system and are significantly associated with multiple diseases. Given that tonsils are located between the oral cavity and laryngoesophagus at the gateway of both alimentary and respiratory tracts, tonsillar tissue may also be affected by both oral (saliva) and alimentary tract microbiota. While several independent reports on oral microbiomes from saliva and tonsils are documented in the literature, limited studies have compared tonsil and saliva microbiota compositions. Here, we analyzed the distribution of the microbial communities in saliva and tonsils of healthy Korean children subjected to tonsillectomy.

\section{Results}

In total, 29 study subjects were enrolled from an initial 45 participants subjected to tonsillectomy owing to hyperplasia over two years. The microbiome profiles of saliva and tonsils were established via $16 \mathrm{~S}$ rRNA gene sequencing. Based on the Chao1 diversity index, the tonsil group showed higher richness in species, compared to the saliva group. On the other hand, the Shannon index revealed a significantly higher biodiversity value for saliva relative to tonsils. The microbial communities of the two groups were highly similar. The top 10 ranked taxa common to both groups determined based on average relative abundance were as follows: Haemophilus, Fusobacterium, Streptococcus, Veillonella, Prevotella 7, Alloprevotella, Porphyromonas, Treponema 2, Campylobacter, and Neisseria. Spearman's rank correlation analysis conducted to elucidate interactions between the groups showed the strongest positive correlation of Treponema 2 between saliva and tonsils. Streptococcus, Veillonella, and Alloprevotella in saliva were negatively correlated with Prevotella 7 in tonsils while Prevotella 7 in saliva showed a strong positive correlation with Alloprevotella in tonsils.

\section{Conclusions}

To our knowledge, this is the first study to present a correlation between tonsil and salivary microbiomes in Korean children who have undergone tonsillectomy. Overall, strong similarities between the tonsil and saliva microbial communities are evident in terms of diversity and composition. Furthermore, the saliva microbiome is expected to significantly affect the tonsil microbiome. Based on the collective results, we propose that the identified microbes act as both intracellular and external environmental factors with a major impact on the functioning of tonsils and their primary cells.

\section{Background}

Human tonsils are lymphoid epithelial tissues of oral mucosa around the oropharynx and nasopharynx that protect the body from pathogen invasion through the mouth and nose. Tonsils are a component of immune system that develop from 2 years of age and grow until puberty, and gradually undergo atrophy thereafter. More frequently in children than in adults, the tonsils can become abnormally enlarged 
(adenotonsillar hyperplasia) or inflamed (tonsillitis), which can be surgically removed by tonsillectomy. Abnormally enlarged tonsillar tissue of pediatric patients is generally caused by hypertrophy rather than inflammation. Hyperplasia of tonsils is a cause of obstructive sleep apnea and recurrent infection of the upper airways. The most common pediatric operative procedure for tonsillar hyperplasia is tonsillectomy [1].

Oral bacteria travel throughout the body and are significantly associated with human diseases. Approximately $8 \times 10^{11}$ bacterial cells per day flow from the mouth to the alimentary tract, and several studies have reported associations of oral microbes between these regions [2-4]. Olsen et al. [5] reported that orally ingested Porphyromonas gingivalis affects intestinal microflora and a variety of oral microbes reach the gut microbiota, but only a subset of these bacteria colonize the gut under dysbiotic conditions. Microbiome analysis at 18 body sites of 200 individuals by Huse et al. [6] showed that core operational taxonomic units (OTUs) shared by more than $95 \%$ subjects were the most abundant in the oral cavity relative to the rest of the body.

Significant quantities of pathogenic bacteria are present in hyperplastic tonsils and adenoids, compared to their normal controls [7-11]. An investigation by Kielmovitch et al. [12] on the tonsillar surface and core of children with recurrent tonsillitis and tonsillar hypertrophy identified Haemophilus influenzae and Bacteroides melaninogenicus as the most prevalent bacteria. Jeong et al. [13] examined the differences between bacterial pathogen constituents of recurrent tonsillitis and tonsillar hypertrophy using the cultivation method in relation to age, season, and antibiotic sensitivity in patients subjected to tonsillectomy. Haemophilus influenzae, Streptococcus pyogenes, Staphylococcus aureus, and Streptococcus pneumoniae were isolated as representative abundant microbes in tonsils.

Until recently, microbiomes have been analyzed using cultivation and PCR methods. Brook et al. [14] performed a microbiological study on the core of tonsils from 50 children with recurrent tonsillitis using the cultivation method. The predominant isolated microbes were Staphylococcus aureus, Moraxella catarrhalis, Peptostreptococcus, pigmented Prevotella and Porphyromonas, and Fusobacterium species. However, culture-based studies present a potentially biased assessment of microbial diversity due to targeting of limited bacterial groups. More recently, 16S rRNA gene-based pyrosequencing and cultureindependent techniques have been applied to survey bacterial communities.

Given that tonsils are located between the oral cavity and laryngoesophagus at the gateway of alimentary and respiratory tracts, the tonsil lymphoid system with antigen capture $\mathrm{M}$ cells similar to those of Peyer's patches of the gut is among the first handling sites for microbial agents and antigens in the human body. The microbial community of saliva can therefore affect that of tonsils and vice versa. To date, oral microbiome studies have mainly targeted saliva samples. Moreover, the majority of tonsil microbiota studies have focused on acute inflammatory disease and not normal conditions. Since deeply branched crypts of the tonsillar epithelium provide optimal spaces for microbiota colonization, it is speculated that the tonsil microbiome has distinct properties from other oral microbiomes. However, no 
studies have examined this theory and further investigation of the correlation between the microbiomes of saliva and tonsils is warranted.

Here, we focused on the distribution and correlations of microbiota in saliva and tonsillar tissues of young tonsillectomy patients based on evaluation of the V3-V5 region of 16S rRNA genes to examine the hypothesis that the microbiome is associated with tonsillar hyperplasia in children. To our knowledge, this is the first comparative analysis of microbiomic portraits in tonsils and saliva of children subjected to tonsillectomy in South Korea. Elucidation of the microbiome constituents of saliva and tonsils in pediatric cases with tonsillar hypertrophy should aid in the treatment of oral disease and development of therapeutic agents.

\section{Results}

\section{General characteristics and biochemical indices of subjects}

A total of 29 healthy participants ( 23 boys and 6 girls) who had undergone tonsillectomy were enrolled. Table 1 presents the general and biochemical characteristics of the participants (left panel) along with the corresponding reference values for Korean children (right panel) $[15,16]$. The average anthropometric measurements were $7.66 \pm 27.78$ years for age, $128.52 \pm 16.84 \mathrm{~cm}$ for height, $32.57 \pm 16.28 \mathrm{~kg}$ for weight, and $18.59 \pm 4.20 \mathrm{~kg} / \mathrm{m}^{2}$ for body mass index (BMI). Eleven participants were $>85$ percentile of $\mathrm{BMI}$ and 18 were $<85$ percentile of BMI. Thus, $37.9 \%$ participants were considered obese and the remaining $62.1 \%$ belonged to the normal weight group. Average values of the biochemical indices from all participants were in the normal Korean pediatric range. 
Table 1

General characteristics and biochemical indices of subjects.

\begin{tabular}{|c|c|c|c|c|c|c|c|}
\hline \multirow{3}{*}{$\begin{array}{l}\text { Parameters } \\
\text { Anthropometric } \\
\text { Measurement }\end{array}$} & \multirow[b]{2}{*}{ Boys } & \multicolumn{3}{|c|}{ Total (Mean \pm S.D.) } & \multicolumn{3}{|c|}{ Korean Pediatrics } \\
\hline & & & 23 & & & & \\
\hline & Girls & & 6 & & & & \\
\hline & Age (years) & 7.66 & \pm & 2.78 & 6 & $\sim$ & 12 \\
\hline & Height (cm) & 128.52 & \pm & 16.84 & 114.7 & $\sim$ & 151.7 \\
\hline & Weight (kg) & 32.57 & \pm & 16.28 & 20.7 & $\sim$ & 45.7 \\
\hline & BMI $\left(\mathrm{kg} / \mathrm{m}^{2}\right)$ & 18.59 & \pm & 4.20 & 15.8 & $\sim$ & 19.8 \\
\hline & $>85$ th percentile & \multicolumn{6}{|c|}{$11(37.9 \%)$} \\
\hline & $<85$ th percentile & \multicolumn{6}{|c|}{$18(62.1 \%)$} \\
\hline \multirow{7}{*}{$\begin{array}{l}\text { Biochemical } \\
\text { Indices }\end{array}$} & Blood Sugar (mg/dL) & 91.72 & \pm & 12.57 & 70 & $\sim$ & 115 \\
\hline & $\begin{array}{l}\text { Total Cholesterol } \\
\text { (mg/dL) }\end{array}$ & 166.67 & \pm & 26.90 & $<170$ & & \\
\hline & AST (U/L) & 24.84 & \pm & 10.84 & \multicolumn{3}{|c|}{$\begin{array}{l}15 \sim 40 \text { (Boys), } 13 \sim \\
35 \text { (Girls) }\end{array}$} \\
\hline & ALT (U/L) & 17.36 & \pm & 10.68 & \multicolumn{3}{|c|}{$\begin{array}{l}10 \sim 35 \text { (Boys), } 10 \sim \\
30 \text { (Girls) }\end{array}$} \\
\hline & WBC $\left(\times 10^{3} / \mathrm{ml}\right)$ & 7.21 & \pm & 2.34 & 4.5 & $\sim$ & 13.5 \\
\hline & $\operatorname{RBC}\left(\times 10^{6} / \mathrm{ml}\right)$ & 4.70 & \pm & 0.26 & 4.2 & $\sim$ & 6.0 \\
\hline & $\mathrm{PLT}\left(\times 10^{3} / \mathrm{ml}\right)$ & 299.16 & \pm & 57.56 & 150 & $\sim$ & 350 \\
\hline
\end{tabular}

Total number of identified and classified microbes in saliva and tonsils

For comparative metagenomic analysis of the microbiomes between saliva and tonsil samples, DNA was extracted and 16S rRNA gene sequencing performed. Sequencing data were used to compare microbial communities between saliva and tonsil groups. A total of 726,274 raw reads were generated from saliva and tonsillar tissue of 29 participants, with an average of 12,745 reads (standard deviation 3,859). The taxonomic compositions of microbiota in saliva and tonsillar tissue were assigned based on the taxonomic level (Table 2). For taxon-based approaches, the sequences were identified OTUs. In the saliva group, 22 phyla, 44 classes, 80 orders, 133 families, 334 genera, 533 species and 1,678 OTUs were annotated while 16 phyla, 28 classes, 44 orders, 73 families, 168 genera, 281 species, and 1,461 OTUs were annotated in the tonsil group. 
Table 2

Analysis of microbiomes between saliva and tonsillar tissues.

\begin{tabular}{|lccccccc|}
\hline $\begin{array}{l}\text { Total number of microbes } \\
\text { identified }\end{array}$ & Phylum & Class & Order & Family & Genus & Species & OTUs \\
\hline Saliva & 22 & 44 & 80 & 133 & 334 & 533 & 1,678 \\
\hline Tonsil & 16 & 28 & 44 & 73 & 168 & 281 & 1,461 \\
\hline
\end{tabular}

\section{Microbiome composition at the genus level in saliva and tonsils}

The microbiome compositions of saliva and tonsillar tissues were determined based on average relative abundance assigned to the phylum and genus levels (Fig. 1). The saliva group showed significantly higher abundance of Firmicutes (45\%), followed by Proteobacteria (23\%), Bacteroidetes (15\%), Actinobacteria (10\%) and Fusobacteria (4\%) at the phylum level, while the tonsil group contained more Proteobacteria (31\%), followed by Bacteroidetes (22\%), Firmicutes (21\%), Proteobacteria (20\%) and Actinobacteria (2\%). At the genus level, Streptococcus (13.4\%) was the predominant taxon in the saliva group and Haemophilus (23.5\%) in the tonsil group.

\section{Comparison Of Alpha Diversities}

We further assessed alpha diversity within each group indicative of species abundance. The alpha diversities of saliva and tonsils were measured based on the Chao1 index and species richness compared. Rarefaction curves for the sequences per sample are shown in Fig. 2a. Chao 1 and Shannon indices were employed to calculate species diversity of samples between saliva and tonsillar groups (Fig. 2b). The Chao1 index values representing microbiome richness in saliva and tonsils showed no significant differences $(p=0.7)$. On the other hand, the Shannon index value representing richness and evenness of the microbial community in saliva was significantly higher than that in tonsil tissue $(p<$ 0.001). Our findings suggest that the bacterial community in saliva is more evenly distributed, compared to that in tonsils.

\section{Principal-coordinates Analysis (pcoa) Biplots}

Microbe communities from saliva and tonsillar tissues were discriminated based on principal coordinates analysis (PCoA). PCoA was used to compare community phylogenetic compositions and revealed microbial diversity between the groups. Based on PCo2 scores, tonsillar tissues were clearly distinguishable from saliva samples (Fig. 3a). A Venn diagram illustrated 334 genera in the saliva group and 168 in the tonsil group (Fig. 3b). Detailed information on common genera between the groups (saliva-specific and tonsil-specific) is listed in Additional file 1. In total, 147 common genera were identified in both sample groups, corresponding to $44.1 \%$ and $87.5 \%$ of the 334 and 187 microbial genera determined in saliva and tonsils, respectively. Overall, $87.5 \%$ of the tonsil microbiome was identical to the 
oral saliva microbiome at the genus level, clearly signifying high similarities of the microbial communities of the two groups.

To determine bacterial distribution from the Venn diagram, a bar graph of all genera at the phylum level was generated (Fig. 3c). Firmicutes, Proteobacteria, Actinobacteria, Bacteroidetes, and Cyanobacteria were most abundant in saliva (upper panel) while in tonsillar tissue, the most abundant genera were Firmicutes, Proteobacteria, Actinobacteria, Bacteroidetes, Cyanobacteria and Fusobacteria (lower panel). Common genera in both tissue types were Firmicutes, Proteobacteria and Actinobacteria and Bacteroidetes (middle panel).

\section{Metastats analysis of differential abundance of top 10 bacterial taxa in saliva and tonsillar samples}

The top 10 ranked taxa in saliva and tonsillar groups based on average relative abundance were Haemophilus (saliva (S): 9.5\%, tonsils (T): 23.5\%), Fusobacterium (S:3.0\%, T:17.2\%), Streptococcus (S:13.4\%, T:8.3\%), Veillonella (S:8.9\%, T:4.5\%), Prevotella 7 (S:2.9\%, T:5.2\%), Alloprevotella (S:1.5\%, $\mathrm{T}: 4.2 \%$ ), Porphyromonas (S:1.3\%, T:3.5\%), Treponema 2 (S:1.3\%, T:3.2\%), Campylobacter (S:1.4\%, $\mathrm{T}: 2.4 \%$ ), and Neisseria (S:3.3\%, T:1.8\%) (Table 3). The phyla of the top 10 ranked taxa were Proteobacteria, Fusobacteria, Firmicutes, Bacteroidetes, and Spirochaetae. Most identified genera included gram-negative bacteria except Streptococcus. Moreover, 6 out of top 10 ranked taxa were obligate anaerobes. However, the most abundant bacteria in tonsils and saliva were facultative anaerobes, such as Haemophilus and Streptococcus. 
Table 3

Top 10 microbes ranked by Metastats analysis.

\begin{tabular}{|lllllll|}
\hline Order & Phylum & Genus & $\begin{array}{l}\text { Saliva } \\
(\%)\end{array}$ & $\begin{array}{l}\text { Tonsil } \\
(\%)\end{array}$ & $\begin{array}{l}\text { Gram } \\
\text { staining }\end{array}$ & Aerobic/Anaerobic \\
\hline 1 & Proteobacteria & Haemophilus & 9.5 & 23.5 & - & $\begin{array}{l}\text { Facultative } \\
\text { anaerobe }\end{array}$ \\
\hline 2 & Fusobacteria & Fusobacterium & 3 & 17.2 & - & Obligate anaerobe \\
\hline 3 & Firmicutes & Streptococcus & 13.4 & 8.3 & + & $\begin{array}{l}\text { Facultative } \\
\text { anaerobe }\end{array}$ \\
\hline 4 & Firmicutes & Veillonella & 8.9 & 4.5 & - & Obligate anaerobe \\
\hline 5 & Bacteriodetes & Prevotella 7 & 2.9 & 5.2 & - & Obligate anaerobe \\
\hline 7 & Bacteriodetes & Alloprevotella & 1.5 & 4.2 & - & Obligate anaerobe \\
\hline 8 & Bacteriodetes & Porphyromonas & 1.3 & 3.5 & - & Obligate anaerobe \\
\hline 9 & Spirochaetae & Treponema 2 & 1.3 & 3.2 & - & Obligate anaerobe \\
\hline 10 & Proteobacteria & Campylobacter & 1.4 & 2.4 & - & Microaerophilic \\
\hline
\end{tabular}

\section{Correlation Plots Of Individual Microbes In Saliva And Tonsils}

Spearman correlation analysis was applied to compare microbiomes within saliva and tonsil tissues as well as between saliva and tonsils for the top 10 ranked taxa (Fig. 4). In general, the top 10 ranked microbiota within saliva showed high positive correlations with each other (Fig. 4a) while the top 10 ranked microbes within tonsils displayed both positive and negative correlations (Fig. 4b). Haemophilus and Fusobacterium within the tonsil group showed the strongest negative correlation with each other ( $r=$ $-0.72, p<0.001)$. Treponema 2 showed the strongest positive correlation between saliva and tonsils ( $r=$ $0.74, p<0.001$ ) while the strongest negative correlation was found between Prevotella 7 in saliva and Alloprevotella in tonsil tissues $(r=-0.40, p<0.01)$ and between Campylobacter in saliva and Neisseria in tonsils ( $r=-0.37, p<0.01$ ) (Fig. 4c). Additional file 2 presents the correlation coefficient scores and $p$ values within saliva and within tonsil tissues and between saliva and tonsils. Microbes with significant correlations $(p<0.01)$ included Streptococcus, Veillonella, Prevotella 7, Alloprevotella, Treponema 2, and Campylobacter in saliva and Prevotella 7, Alloprevotella, Treponema 2, and Neisseria in tonsils. Prevotella 7 in tonsils showed significant positive correlations with Streptococcus $(r=0.43)$, Veillonella $(r=0.38)$, and Alloprevotella $(r=0.38)$ in saliva. Meanwhile, Prevotella 7 and Campylobacter in saliva showed significant negative correlations with Alloprevotella $(r=-0.40)$ and Neisseria in tonsils $(r=-0.37)$, respectively. 


\section{Taxonomic-level Comparison Of Microbiota In Saliva And Tonsils}

Linear discriminant analysis (LDA) effect size (LEfSe) analysis was used to identify significantly different saliva and tonsil microbiota compositions for the top 10 ranked taxa (Fig. 5). The threshold of the logarithmic LDA score for discriminative features was $>2.5$. Enriched genera in the saliva group were Neisseria, Streptococcus, and Veillonella and those in the tonsil group were Fusobacterium, Haemophilus, Prevotella 7, Alloprevotella, Campylobacter, Porphyromonas, and Treponema 2.

\section{Discussion}

To our knowledge, this is the first study to compare the core microbiomes of saliva and tonsils in Korean pediatric patients without tonsillitis. By employing $16 \mathrm{~s}$ rRNA gene sequencing, we determined the relative abundance of the microbial community in saliva and tonsils, with a view to establishing the association between the oral saliva microbial community and tonsillar microbiome profile in Korean children (Fig. 6). Our data suggest that the microbiome profiles of saliva and tonsils are largely similar (87.5\%), suggestive of interactions between their microbial components. To date, most studies on pediatric tonsils have reported the tonsil microbiomes under inflammatory conditions such as tonsillitis. Our study is distinct from these previous studies, in that we examined saliva and tonsil microbiomes in the absence of tonsillitis. Nevertheless, our study could not elucidate the microbiome of perfectly healthy tonsils, because the tonsil microbiota samples used in this study were obtained from patients diagnosed with tonsillar hypertrophy. However, because children with normal tonsils do not need to undergo tonsillectomy, it is almost impossible to obtain perfectly normal tonsil tissues.

Since tonsils have emerged as an good source of adult stem cells in tissue engineering and regenerative medicine with the added advantages of noninvasiveness of tissue collection [17], relatively high proliferation rate and low allogenicity, our research may facilitate the development of microbiome-based tools to regulate tonsil-derived therapeutic platforms. Further studies on larger groups are warranted to confirm initial findings.

Recent advances in sequencing technology and metagenomics have expanded our knowledge of the composition and association of the oral microbiome with human health and disease. The oral cavity microbiome has generally been examined by collecting oral rinse samples, including saliva, but it remains

to be established whether saliva provides an accurate representation of the microbiome of the oral cavity [18]. Given that the human oral cavity is composed of various subsites, including teeth, gingival sulcus, tongue, hard and soft palates, and tonsils, which provide appropriate space for colonization but slightly different environments for microorganisms, the salivary microbiome profile may not be correlated with those of other subsites of the oral cavity.

Although a number of differences in the microbial profiles between recurrent tonsillitis and tonsillar hypertrophy pediatric groups have been determined by Jeong et al. [13] using culture-based analysis, these experiments were limited in that they only included aerobic bacteria existing in the tonsillar core and many human associated microorganisms are not cultivable under laboratory settings. Accordingly, 
we aimed to elucidate and compare the core microbiome profiles from paired saliva-tonsil samples using metagenome-wide analysis.

We obtained an average of 12,745 raw read counts, which was markedly lower than the 80,829 raw read count average reported by Jensen et al. [19]. However, Caporaso and co-workers [20] demonstrated that 2,000 reads are sufficient to resolve relationships among samples observed with the full dataset. Accordingly, we considered our data adequate to determine microbiome correlations between saliva and tonsils. Our dataset identified from 12,745 reads included 1,678 OTUs in saliva and 1,461 OTUs in tonsils, which were reasonable counts for further analysis.

Alpha-diversity indices, including Chao1 and Shannon-Weaver diversity indicies, are generally used to compare the differences between microbial communities [21-23]. Both saliva and tonsils showed similar richness in microbial communities but the saliva microbiome presented higher evenness than tonsil tissue. The microbiome profile of saliva appears more stable relative to that of tonsils. Saliva is constantly secreted from salivary glands into the oral cavity containing a diverse bacterial population while the microbial community in tonsils is mainly colonized in the recessed epithelium of deeply branched crypts $[18,24]$. Therefore, saliva continuously secreted from various oral sites contains diverse bacteria but the range in tonsils is lower since the microbial populations stagnate in the crypts and remain limited.

Lazarevic et al. [25] identified the main phyla in saliva as Firmicutes, Proteobacteria, Actinobacteria, Bacteroidetes, Fusobacteria, Spirochaetes, and Saccharibacteria. Pyrosequencing analysis of saliva microbes in healthy Chinese children and adults by Ling et al. [26] disclosed Streptococcus, Prevotella, Neisseria, Haemophilus, Porphyromonas, Gemella, Rothia, Granulicatella, Fusobacterium, Actinomyces, Veillonella, and Aggregatibacter as major components of the healthy saliva microbiome. In our study, Streptococcus was the most abundant component, followed by Haemophilus, Veillonella, Neisseria, Fusobacterium, Prevotella 7, and similar to the profile determined by Ling and co-workers [26].

In a study on bacterial distribution in tonsils of Korean children, Jeong et al. [13] isolated 966 microbes from the tonsil cores of 824 recurrent tonsillitis and tonsillar hypertrophy patients using the cultivation method. In their experiments, Haemophilus influenzae (31.4\%) was most commonly isolated from cases of tonsillar hypertrophy, followed by Streptococcus pyogenes (24.2\%), Staphylococcus aureus $(22.9 \%)$ and Streptococcus pneumoniae (12.6\%). Similar to these earlier findings, Haemophilus influenzae was the dominant pathogen in the tonsillar hypertrophy group in our study. Jensen and colleagues [19] compared the microbiomes of tonsillar crypts in children and adults affected by recurrent tonsillitis with those of healthy adults and children with tonsillar hyperplasia. Twelve genera of microbial communities were identified in all samples regardless of age and health status. Notably, Haemophilus influenzae, Neisseria species and Streptococcus pseudopneumoniae were significantly more abundant in children. In our experiments, Haemophilus (23.5\%) was the most prevalent bacterial species in tonsil tissue, followed by Fusobacterium (17.2\%) and Streptococcus (8.3\%). Haemophilus has thus been identified as the most common bacterium, not only in recurrent tonsillitis but also hypertrophic tonsil samples $[9,13,27,28]$. 
Haemophilus and Staphylococcus are the representative microbiota in both tonsillectomized and nontonsillectomized children with recurrent and tonsillar hypertrophy [27, 28]. Haemophilus was identified as the most common bacterial species in both saliva and tonsils in our study (Table 3). However, Staphylococcus, which is frequently detected in tonsillitis, was less abundant in our study and weakly correlated between the groups (data not shown). Specifically, Staphylococcus was ranked as 22 in terms of abundance in tonsils and 38 in saliva. The low abundance of Staphylococcus may be explained by the fact that the bacterium is mainly associated with inflammation and we selected only hyperplasia cases for analysis (except tonsillitis).

According to Kuhn et al. [10], the predominant aerobic and facultative organisms were Haemophilus influenzae, followed by Neisseria and Staphylococcus aureus, in pediatric patients subjected to tonsillectomy, while the predominant anaerobic bacteria were Fusobacterium, Bacteroides and Prevotella melaninogenica. These earlier results were identical to our finding that Haemophilus, Fusobacterium, Prevotella, and Neisseria are among the top 10 ranked genera in tonsil and saliva samples of Korean children (Table 3). Among the top 10 microbiota identified in the current study, the dominant bacteria in both saliva and tonsils were facultative anaerobes. Moreover, the most abundant species in saliva (Streptococcus of Firmicutes phylum) and tonsils (Haemophilus of Proteobacteria phylum) were consistent with previous reports $[10,25,26,29]$.

The top 10 ranked microbes in our study mostly showed a strong positive correlation within the saliva microbiome while both positive and negative correlations were detected within the tonsil microbiome. These differences could be explained as follows: since saliva is continuously secreted and renewed in the oral cavity, the microbial community is relatively constant. Compared with saliva, microbiota in tonsils are exposed to an environment with insufficient supply of oxygen and nutrients due to stagnation in the crypt, potentially leading to competition between two microbes and, consequently, negative correlation.

Treponema 2 showed the most significant positive correlation between saliva and tonsil environments (Fig. 4), although its abundance was low (1.3\% in saliva, 3.2\% in tonsils; Table 3 ). The genus Treponema contains both pathogenic (treponematoses) and non-pathogenic species. Non-pathogenic treponemes form part of the normal microbial flora of the oral cavity, intestinal tract or genital tract. A number of oral treponemes have been associated with gingivitis and periodontal disease [30]. Prevotella 7 in tonsils was positively correlated with Streptococcus, Veillonella, and Alloprevotella in saliva. Prevotella 7 is one of the major periodontal disease- causing microbiota, such as periodontitis and gingivitis. Furthermore, Streptococcus in saliva, which affects Prevotella 7 in tonsils, triggers airway infections, such as pharyngitis, tonsillitis, and tympanitis. This strong positive correlation supports a link between dental inflammatory and oropharyngeal inflammatory diseases, further indicating a significant connection between the oral cavity (saliva) and oropharynx (tonsil).

Based on the collective results, we have expanded our understanding of the interactions between microbiomes of saliva and tonsils. Several factors are responsible for loss of microbial diversity and homeostatic function, including inflammation, diet, xenobiotics, and altered host cell function [31]. Given 
its critical roles in multiple diseases, the microbiome has become an extremely attractive target for therapeutic interventions [32]. One of the biggest hurdles in microbiome research is identification of cause-effect relationships and designing of microbiome-based therapies that are able to achieve predictable outcomes on the microbial community and host cell function. Further studies are required to develop strategies aimed at modulating the microbiome profile for improvement of host function and health.

\section{Conclusions}

Analysis of the microbiomes of paired tonsil and saliva samples from Korean pediatric patients diagnosed with hyperplasia without tonsillitis revealed that many bacterial communities are shared and show similarities in terms of diversity and composition, suggesting close interactions between the two microbial groups. Although further studies are clearly essential, we assume that the oral microbiome exerts significant effects on not only the tonsil itself but also tonsil-derived immune or stem cells through regulating the microbial community. Our study serves as a cornerstone of tonsillar microbiome research and the data obtained should facilitate the development of novel microbiome-based strategies to regulate tonsil-derived therapeutic platforms, such as immune or stem cell therapy.

\section{Methods}

\section{Sample collection}

Among the 45 participants who underwent tonsillectomy at Chungnam National University Hospital (CNUH, Daejeon, Korea) between June 2018 and January 2019, 29 were enrolled for analysis. Inclusion criteria were: (a) ages younger than 10 years with benign tonsillar hypertrophy > Friedman grading scale 2 resulting in sleep apnea and/or mouth breathing and (b) non-denture or brace wearers. Exclusion criteria were: (a) recent (last 3 months) or frequent (3 times/year) history of tonsillitis, (b) systemic disease, (c) intake of antibiotics in the last 3 months, (d) a habit of using oral rinse regularly and (e) breastfeeding.

Oral examination and sample collection were conducted at the Department of Otolaryngology-Head and Neck Surgery of CNUH. All participants remained fasted after midnight the day before until completion of surgery according to the regular preoperative protocol.

Saliva was collected from mouths of patients on the morning of surgery. Prior to sample collection, participants were instructed not to brush their teeth from the previous night to the time of sampling and prohibited from eating or drinking for at least 9 hours. In brief, participants were asked to chew on sterile gauze ( $4 \times 4$ inch) for 1 minute to stimulate salivation before placing the gauze with absorbed saliva back into a sterile Eppendorf conical tube $(50 \mathrm{ml})$. Tubes were centrifuged for 15 minutes at 1,763 $\mathrm{g}$ with a $100 \mu \mathrm{m}$ cell strainer and used to collect clear saliva samples that were (immediately) stored at $-70{ }^{\circ} \mathrm{C}$ until DNA extraction. 
After general anesthesia, en-bloc resection of both palatine tonsils via the peroral approach was performed according to the traditional tonsillectomy procedure using a cold knife and electrocautery. About half the tissue around the upper pole of palatine tonsils was sent to a pathological laboratory for clinical diagnosis and the remainder used for microbiome analysis.

\section{Anthropometric Measurements And Serum Biochemical Indices Of Subjects}

We collected anthropometric data from all subjects, including height and weight of all patients, which were measured the day before surgery. Body mass index (BMI) was calculated according to a previous report [33]. In addition, with reference to the 2017 Korean National Growth Chart, subjects were divided into two groups based on BMI 85th percentile to determine obesity status.

Serum biochemical indices including blood glucose, total cholesterol, liver enzymes such as Aspartate aminotransferase (AST), Alanine aminotransferase (ALT), hite blood cell (WBC), red blood cell (RBC), and platelet (PLT) counts, were acquired from routine preoperative laboratory tests according to the guidelines of American Society of Anesthesiologists (ASA).

\section{DNA Extraction And Amplicon Sequencing}

DNA from saliva and tonsillar tissue was extracted using a DNeasy PowerSoil kit (QIAGEN, Germany). DNA in each sample was quantified using QIAxpert (QIAGEN, Germany). Specific V3-V4 hypervariable regions of the 16S rRNA gene were amplified using 16S_V3_F (5'-

TCGTCGGCAGCGTCAGATGTGTATAAGAGACAGCCTACGGGNGG CWGCAG - 3') and 16S_V4_R (5'GTCTCGTGGGCTCGGAGATGTGTATAAGAGACA GGACTACHVGGGTATCTAATCC - 3') primers. Libraries were prepared using PCR products and quantified with the aid of the QIAxpert kit (QIAGEN). Each amplicon was quantified, pooled, and sequenced using MiSeq (Illumina, USA).

\section{Analysis Of Bacterial Compositions Of Microbiomes}

Paired-end reads matching the adapter sequences were trimmed using cutadapt (version 1.1.6) [34]. The resulting FASTQ files containing paired-end reads were merged with CASPER and quality-filtered with Phred (Q) score of 20 [35]. Any reads $<350 \mathrm{bp}$ or $>550 \mathrm{bp}$ after merging were additionally excluded. To identify the chimeric sequences, a reference-based chimera detection step was conducted with VSEARCH against the SILVA gold database [36, 37]. Next, sequence reads were clustered into OTUs using VSEARCH with a de novo clustering algorithm under $97 \%$ sequence similarity. The representative sequences of OTUs were finally classified using SILVA 128 database with UCLUST (script on QIIME version 1.9.1) under default parameters [38].

\section{Statistical analysis}

Alpha diversities were calculated on a rarified dataset $(6,039$ reads for saliva and 8,031 reads for tonsillar tissue) using the QIIME pipeline. The alpha diversities for species richness and evenness (Chao1 index and Shannon index) were calculated. Statistical comparison of alpha diversities between tonsils and saliva was performed using the Kruskal-Wallis test and $p$ values adjusted with Benjamini-Hochberg 
correction. The resultant distance matrix was applied to generate principal coordinate analysis (PCoA) plots based on Bray-Curtis distance. The top 10 microbes ranked in terms of abundance were selected via Metastats analysis [39]. Wilcoxon rank-sum test was additionally performed to compare the relative abundance of microbial taxa between saliva and tonsil groups.

Correlations of genera between saliva and tonsil groups were further analyzed using Spearman's correlation coefficient. To identify microbial taxa, linear discriminant analysis (LDA) effect size (LEfSe) considering statistical significance and biological relevance was performed. The threshold of microbial taxa was identified as LDA score $>2.5$. All statistical analyses were performed using $\mathrm{R}$ version 3.6.1. The $p$ values $<0.05$ were considered statistically significant.

\section{Declarations}

\section{Ethics approval and consent to participate}

This study was given ethical approval by the Forum for Ethical Review Committees in Asia and the Western Pacific Region (FERCAP) certified institutional review board (IRB) of Chungnam National University Hospital (Daejeon, Korea, IRB approval number: CNUH 2018-06-021-002). All participants understood the purpose of the study and provided informed consent. All experiments were performed in accordance with approved guidelines.

\section{Consent for publication}

Not applicable.

\section{Availability of data and materials}

All raw sequences derived from this experiment were submitted into the Short Read Archive of NCBI and can be found under the BioProject accession number \#PRJNA615768

(https://www.ncbi.nlm.nih.gov/sra/PRJNA615768). Other generated and analyzed data are included in this publication.

\section{Competing interests}

All authors have approved the final version of the manuscript. The authors declare that they have no conflicts of interest to disclose.

\section{Funding}

This study was supported by the National Research Foundation of Korea (NRF) grants funded by the Korean government (MEST) (Grant numbers: NRF-2016R1D1A1B04932112) and by the Ministry of Science, ICT \& Future Planning (No. 2016M3A9B4919639, 2017R1A2B4002611, 2019M3A9H1032376).

\section{Authors' contributions}


DHC, JKC, and KEL performed the experimental work. JWP drafted the manuscript. WHL and JHY performed the bioinformatics. JYL and YJP designed the experimental and analyzed the data. BSK and CO collected the samples. HRW manged IRB. JWC designed the experimental and contributed to the writing of the manuscript. YSP designed the series of experiments. All authors read and approved the final manuscript.

\section{Acknowledgements}

Not applicable.

\section{Author information}

${ }^{1}$ Major in Microbiology, School of Biological Sciences, College of Natural Sciences, Chungbuk National University, Cheongju 28644, Republic of Korea. ${ }^{2}$ Institute of MD Healthcare Inc, Seoul, Republic of Korea.

${ }^{3}$ Central Research Institute, Nano Intelligent Biomedical Engineering Corporation (NIBEC), School of Dentistry, Seoul National University, Seoul 03080, Republic of Korea. ${ }^{4}$ Department of Dental Regenerative Bioengineering and Dental Research Institute, School of Dentistry, Seoul National University, Seoul 03080, Republic of Korea. ${ }^{5}$ Department of Otolaryngology-Head and Neck Surgery, Chungnam National University College of Medicine, Daejeon 35015, Republic of Korea

\section{References}

1. Caers $G$, Hensgens $C$. Antimicrobial and surgical treatment of tonsils. Bacteriological and histological data collected on 44 tonsillectomized children. Acta Otorhinolaryngol Belg. 1977;31:554-65.

2. Curtis MA, Zenobia C, Darveau RP. The relationship of the oral microbiotia to periodontal health and disease. Cell Host Microbe. 2011;10:302-6.

3. Gao L, Xu T, Huang G, Jiang S, Gu Y, Chen F. Oral microbiomes: more and more importance in oral cavity and whole body. Protein Cell. 2018;9:488-500.

4. Segata N, Haake SK, Mannon P, Lemon KP, Waldron L, Gevers D, Huttenhower C, Izard J. Composition of the adult digestive tract bacterial microbiome based on seven mouth surfaces, tonsils, throat and stool samples. Genome Biol. 2012;13:R42.

5. Olsen I, Yamazaki K. Can oral bacteria affect the microbiome of the gut?. J Oral Microbiol. 2019;11.

6. Huse SM, Ye Y, Zhou Y, Fodor AA. A core human microbiome as viewed through $16 \mathrm{~S}$ rRNA sequence clusters. PLoS One. 2012;7:1-12.

7. Dedio RM, Tom LWC, Mcgowan KL, Wetmore RF, Handler SD, Potsic WP. Microbiology of the Tonsils and Adenoids in a Pediatric Population. Arch Otolaryngol Neck Surg. 1988;114:763-5.

8. Brodsky L, Moore LSJ. The role of haemophilus influenzae in the pathogenesis of tonsillar hypertrophy in children. Laryngoscope. 1988;98:1055-60. 
9. François M, Bingen E, Soussi T, Narcy P. Bacteriology of tonsils in children: comparison between recurrent acute tonsillitis and tonsillar hypertrophy. Adv Otorhinolaryngol. 1992;47:146-50.

10. Kuhn JJ, Church LWP, Brook I, Bianchi DA, Waters CL, Thompson DH. Quantitative bacteriology of tonsils removed from children with tonsillitis hypertrophy and recurrent tonsillitis with and without hypertrophy. Ann Otol Rhinol Laryngol. 1995;104:646-52.

11. Surow JB, Handler SD, Telian SA, Fleisher GR, Baranak CC. Bacteriology of Tonsil Surface and Core in Children. Laryngoscope. 1989;99:261-6.

12. Kielmovitch IH, Keleti G, Bluestone CD, Wald ER, Gonzalez C. Microbiology of Obstructive Tonsillar Hypertrophy and Recurrent Tonsillitis. Arch Otolaryngol Neck Surg. 1989;115:721-4.

13. Jeong JH, Lee DW, Ryu RA, Lee YS, Lee SH, Kang JO, Tae K. Bacteriologic comparison of tonsil core in recurrent tonsillitis and tonsillar hypertrophy. Laryngoscope. 2007;117:2146-51.

14. Brook I, Yocum P, Foote PA. Changes in the core tonsillar bacteriology of recurrent tonsillitis: 19771993. Clin Infect Dis. 1995;21:171-6.

15. Nah EH, Kim S, Cho S, Cho HI. Complete Blood Count Reference Intervals and Patterns of Changes Across Pediatric, Adult, and Geriatric Ages in Korea. Ann Lab Med. 2018;38:503-11.

16. Cho S-M, Lee S-G, Kim HS, Kim J-H. Establishing pediatric reference intervals for 13 biochemical analytes derived from normal subjects in a pediatric endocrinology clinic in Korea. Clin Biochem. 2014;47:268-71.

17. Oh SY, Choi DH, Jin YM, Yu Y, Kim HY, Kim G, Park YS, Jo I. Optimization of Microenvironments Inducing Differentiation of Tonsil-Derived Mesenchymal Stem Cells into Endothelial Cell-Like Cells. Tissue Eng Regen Med. 2019;16:631-43.

18. Jo R, Nishimoto Y, Umezawa K, Yama K, Aita Y, Ichiba Y, et al. Comparison of oral microbiome profiles in stimulated and unstimulated saliva, tongue, and mouth-rinsed water. Sci Rep. 2019;9:1-7.

19. Jensen A, Fagö-Olsen H, Sørensen CH, Kilian M. Molecular Mapping to Species Level of the Tonsillar Crypt Microbiota Associated with Health and Recurrent Tonsillitis. PLoS One. 2013;8.

20. Caporaso JG, Lauber CL, Walters WA, Berg-Lyons D, Lozupone CA, Turnbaugh PJ, Fierer N, Knight R. Global patterns of $16 S$ rRNA diversity at a depth of millions of sequences per sample. Proc Natl Acad Sci U S A. 2011;108 SUPPL. 1:4516-22.

21. Kim BR, Shin J, Guevarra RB, Lee JH, Kim DW, Seol KH, Lee JH, Kim HB, Isaacson RE. Deciphering diversity indices for a better understanding of microbial communities. J Microbiol Biotechnol. 2017;27:2089-93.

22. Chao A, Bunge J. Estimating the number of species in a stochastic abundance model. Biometrics. 2002;58:531-9.

23. Strong WL. Biased richness and evenness relationships within Shannon-Wiener index values. Ecol Indic. 2016;67:703-13.

24. Yu G, Phillips S, Gail MH, Goedert JJ, Humphrys MS, Ravel J, Ren Y, Caporaso NE. The effect of cigarette smoking on the oral and nasal microbiota. Microbiome. 2017;5:1-6. 
25. Lazarevic V, Whiteson K, Hernandez D, François P, Schrenzel J. Study of inter- and intra-individual variations in the salivary microbiota. BMC Genom. 2010;11:523.

26. Ling Z, Liu X, Wang Y, Li L, Xiang C. Pyrosequencing Analysis of the Salivary Microbiota of Healthy Chinese Children and Adults. Microb Ecol. 2013;65:487-95.

27. Van Staaij BK, Van Den Akker EH, De Haas Van Dorsser EHM, Fleer A, Hoes AW, Schilder AGM. Does the tonsillar surface flora differ in children with and without tonsillar disease? Acta Otolaryngol. 2003;123:873-8.

28. Brodsky L, Moore L, Stanievich J. The role of haemophilus influenzae in the pathogenesis of tonsillar hypertrophy in children. Laryngoscope. 1988;98:1055-60.

29. Taylan I, Özcan I, Mumcuoğlu I, Baran I, Özcan KM, Akdoğan Ö, Selcuk A, Balaban N, Dere H. Comparison of the Surface and Core Bacteria in Tonsillar and Adenoid Tissue With Beta-Lactamase Production. Indian J Otolaryngol Head Neck Surg. 2011;63:223-8.

30. Radolf JD. Treponema. In: Baron. S, editor. Medical Microbiology. 4th editio. Texas: The University of Texas Medical Branch at Galveston.; 1996.

31. Levy M, Kolodziejczyk AA, Thaiss CA, Elinav E. Dysbiosis and the immune system. Nat Rev Immunol. 2017;17:219-32.

32. Thaiss CA, Elinav E. The remedy within: will the microbiome fulfill its therapeutic promise? J Mol Med. 2017;95:1021-7.

33. Deurenberg P, Weststrate JA, Seidell JC. Body mass index as a measure of body fatness: age- and sex-specific prediction formulas. Br J Nutr. 1991;65:105-14.

34. Martin M. Cutadapt removes adapter sequences from high-throughput sequencing reads. EMBnet J. $2011 ; 17: 10-2$.

35. Kwon S, Lee B, Yoon S. CASPER: context-aware scheme for paired-end reads from high-throughput amplicon sequencing. BMC Bioinformatics. 2014;15:1-11.

36. Quast C, Pruesse E, Yilmaz P, Gerken J, Schweer T, Yarza P, Peplies J, Glöckner FO. The SILVA ribosomal RNA gene database project: Improved data processing and web-based tools. Nucleic Acids Res. 2013;41:590-6.

37. Rognes T, Flouri T, Nichols B, Quince C, Mahé F. VSEARCH: A versatile open source tool for metagenomics. PeerJ. 2016;4:1-22.

38. Caporaso JG, Kuczynski J, Stombaugh J, Bittinger K, Bushman FD, Costello EK, et al. QIIME allows analysis of high-throughput community sequencing data. Nat Methods. 2010;7:335-6.

39. White JR, Nagarajan N, Pop M. Statistical Methods for Detecting Differentially Abundant Features in Clinical Metagenomic Samples. PLoS Comput Biol. 2009;5:e1000352.

\section{Figures}


(A)

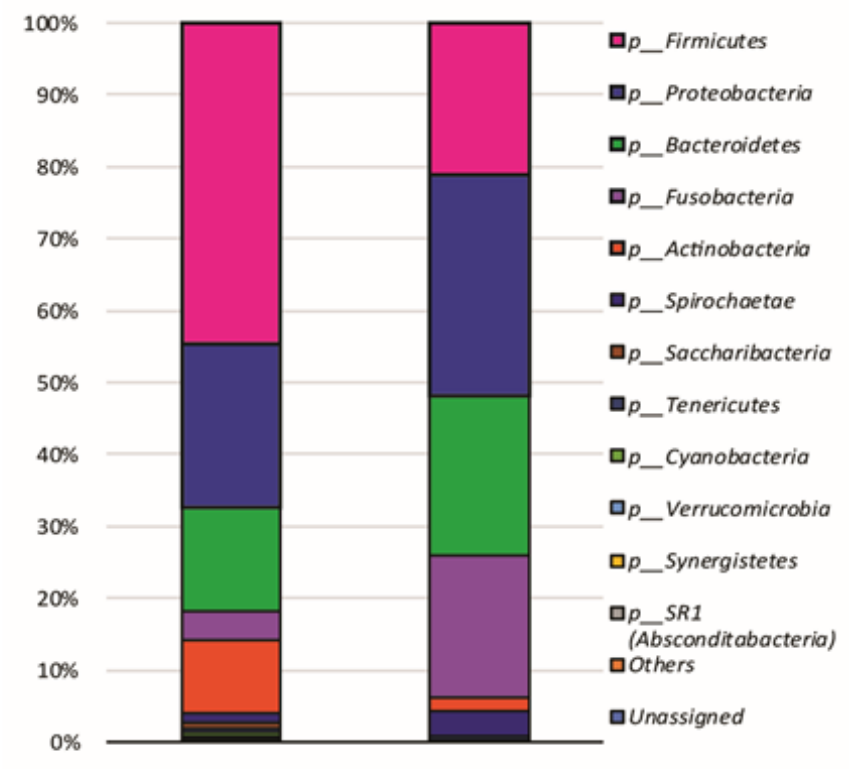

(B)

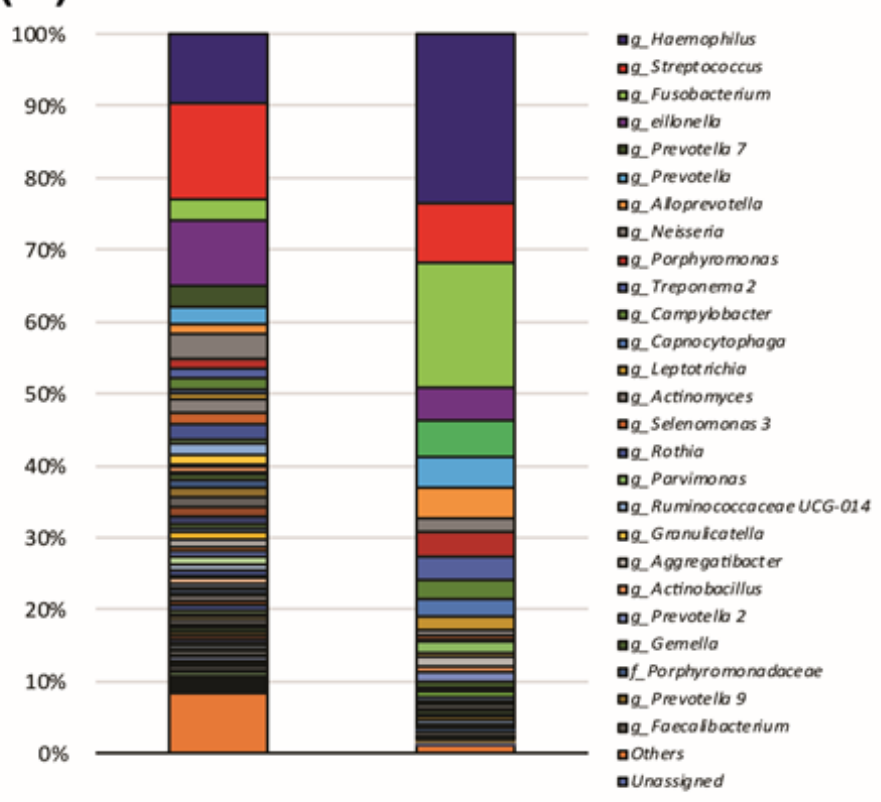

Figure 1

Microbiota compositions of saliva and tonsil samples from 29 participants at the phylum level $(A)$ and genus level (B). Relative abundance of the microbial community based on the dominant phyla and genera. The highly abundant phyla were Firmicutes, Proteobacteria, Bacteroidetes, Fusobacteria, and Actinobacteria. The predominant genera were Haemophilus, Streptococcus, Fusobacterium, Veillonella, and Prevotella 7. 
(A)

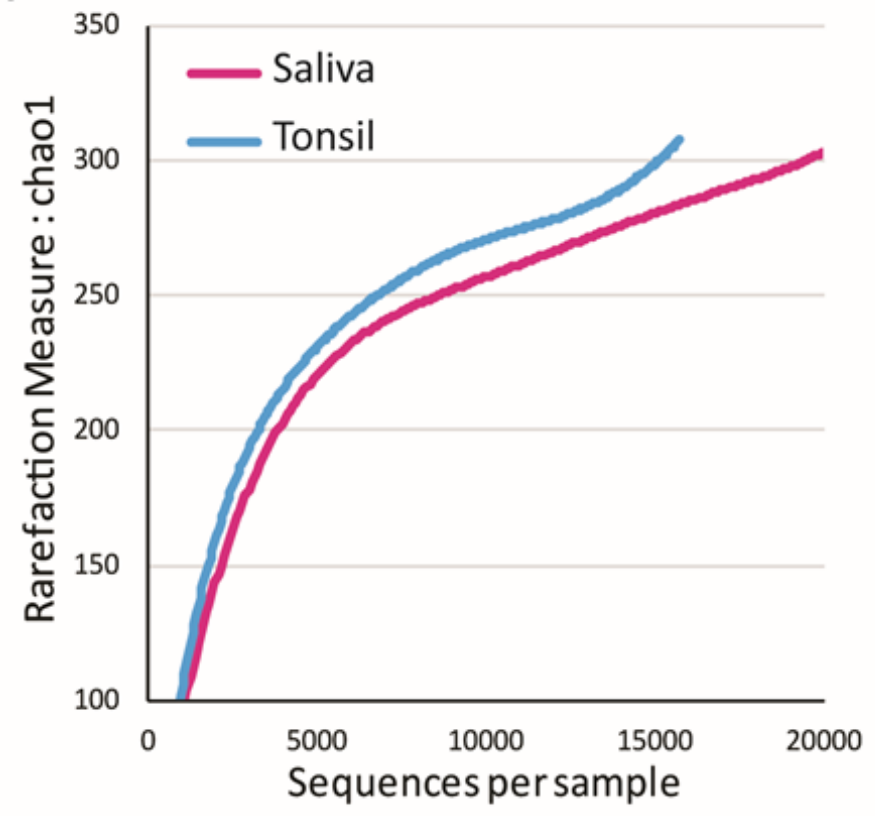

(B)
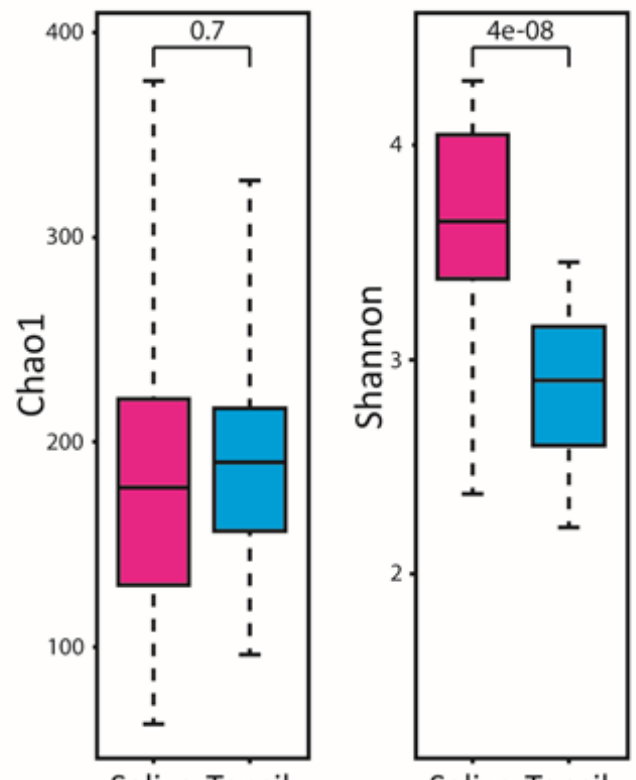

Saliva $\square$ Tonsil

\section{Figure 2}

Comparison of alpha diversity between saliva and tonsils. Rarefaction measured based on Chao1 index (A) and box plots for alpha diversity based on Chao1 and Shannon indices (B). Magenta represents saliva and blue signifies tonsil tissues. 
(A)

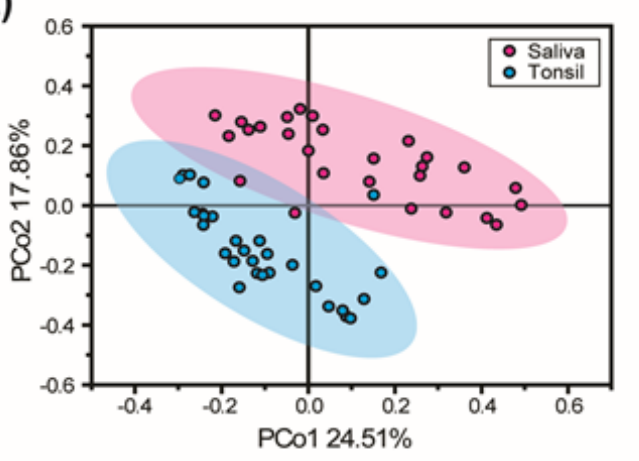

(B)

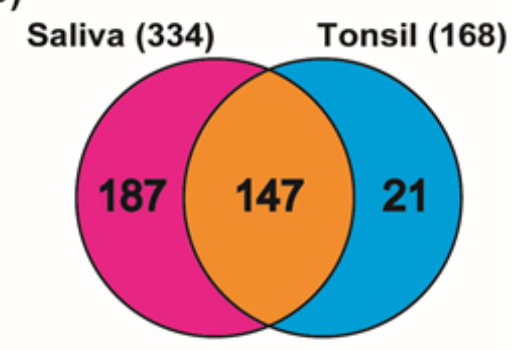

(C)
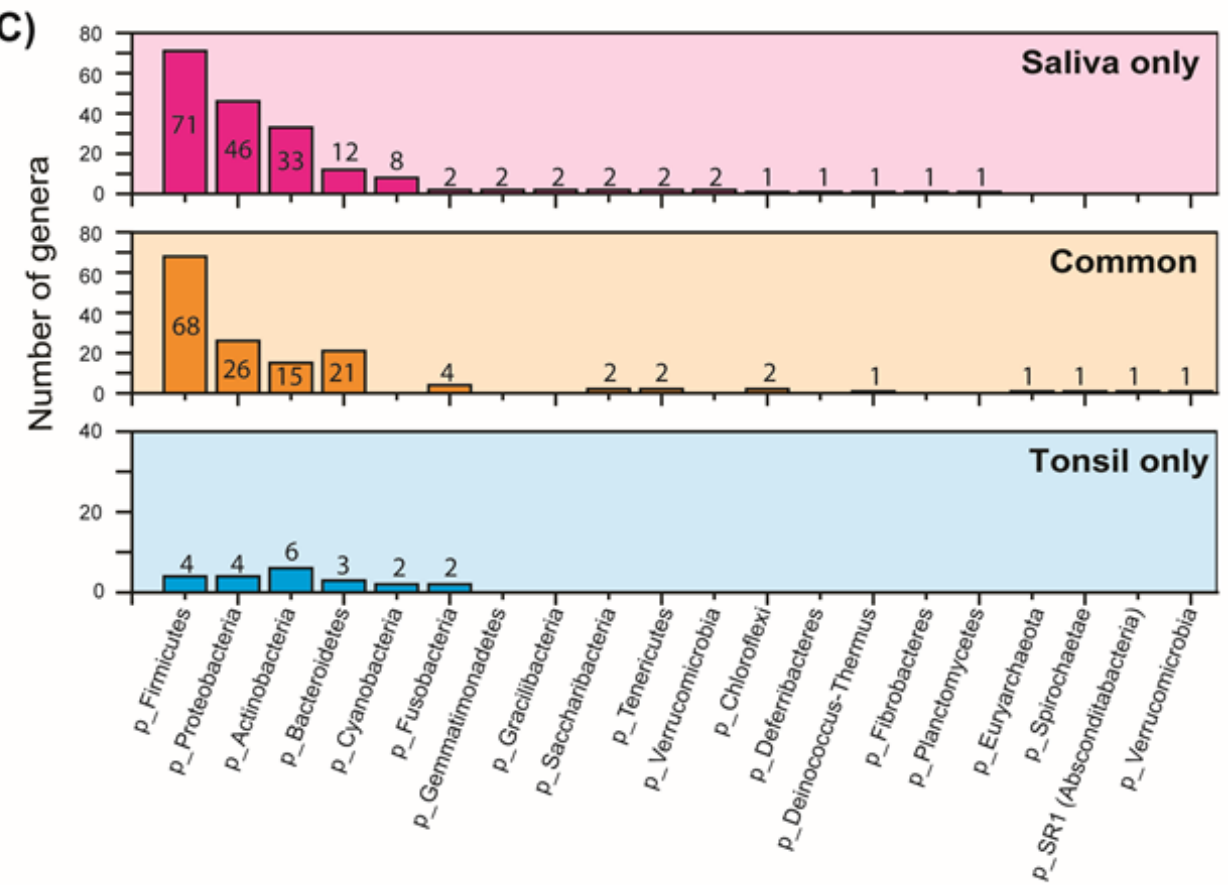

Figure 3

Comparison of saliva and tonsil groups at the genus level. Principal Coordinate Analysis plots of BrayCurtis-computed distances between saliva and tonsils. (A) Saliva (Red) and tonsil (Blue) samples are colored according to subject. The plot presents $95 \%$ confidence ellipse with a color background. (B) Venn diagram showing overall overlap between saliva and tonsils at the genus level. (C) Number of specific and common genera between saliva and tonsil samples at the phylum level. 

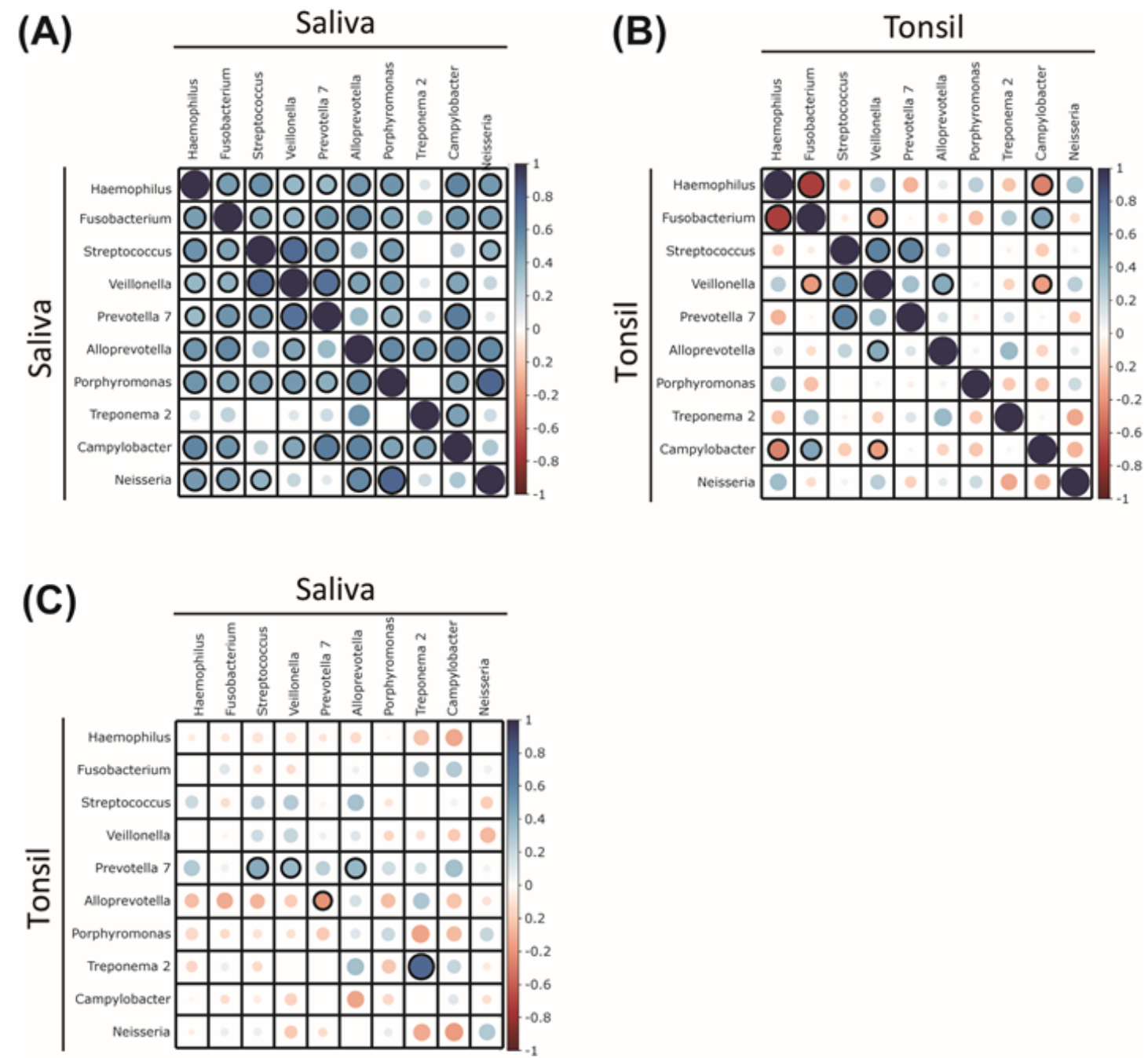

\section{Figure 4}

Correlation of the top 10 ranked microbes. Correlation plots of the top 10 ranked microbes within saliva (A), within tonsil (B), and between saliva and tonsil (C). A correlation matrix plot (based on Spearman's correlation) is presented. Positive and negative correlations are represented by blue or red circles, respectively, and size and color of circles refer to the correlation value. Significant correlations $(P \leq 0.05)$ are indicated by black outline of the circle. 


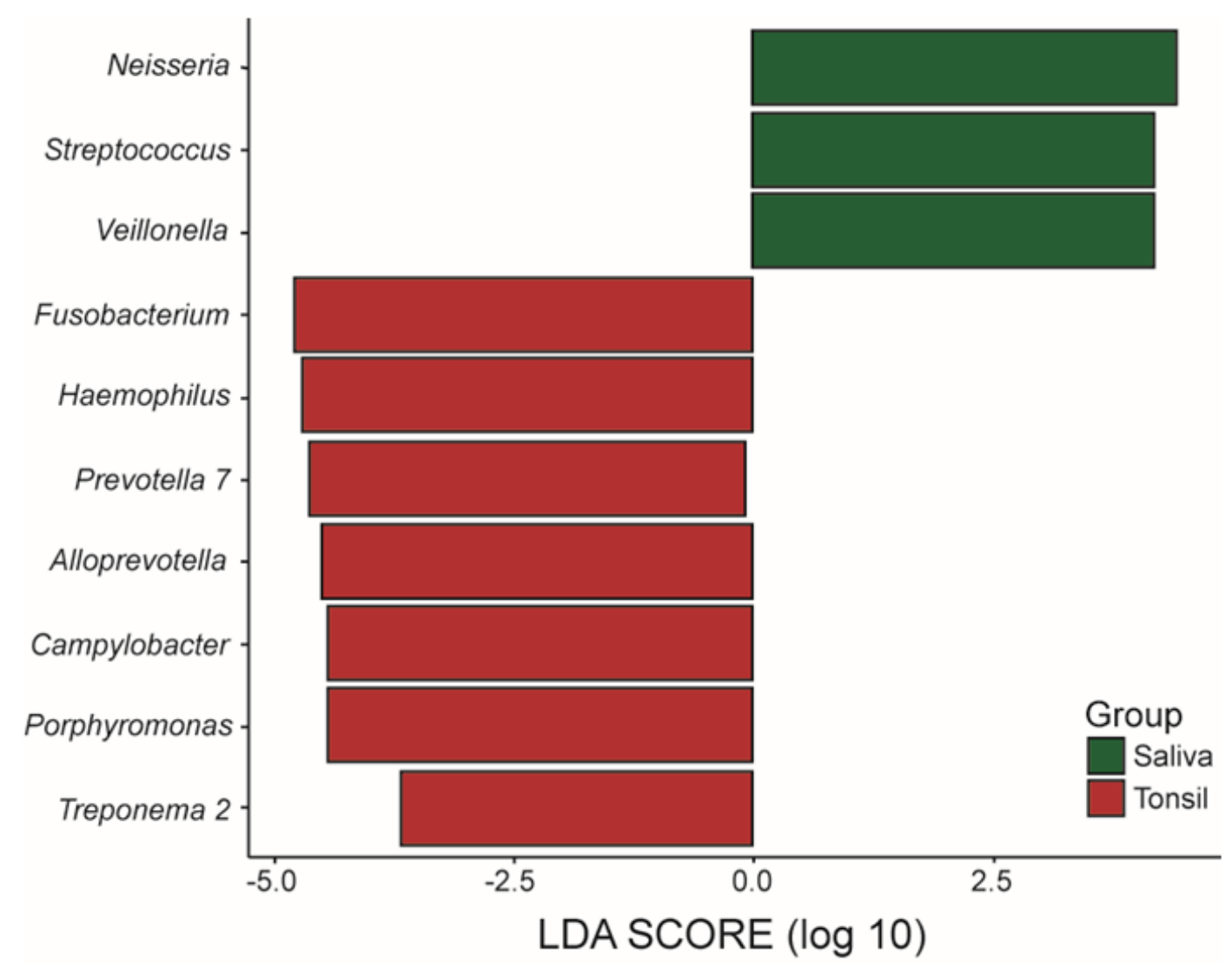

Figure 5

LDA effect size (LEfSe) analysis of tonsils compared to saliva for the top 10 ranked genera. LEfSe analysis used to select microbiota significantly more abundant in one group, while the bar size represents the size of the effect. Green bars represent microbiota associated with saliva while red bars represent tonsil-associated microbiota. 


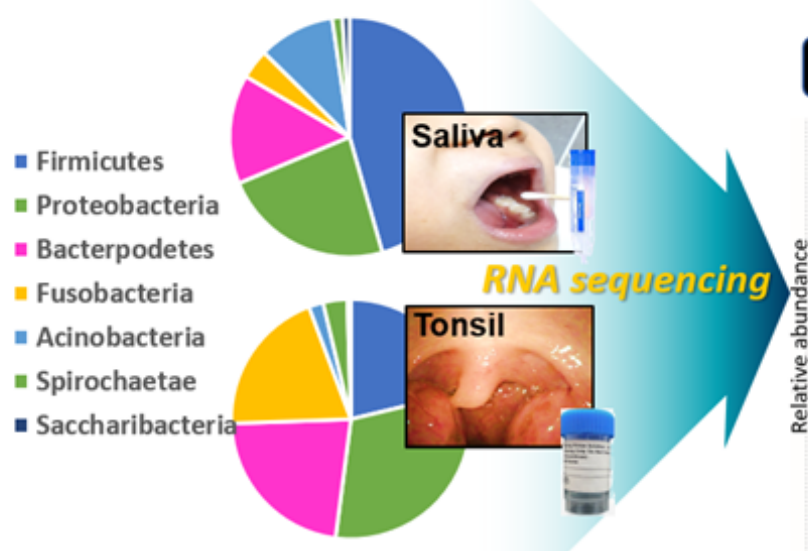

\section{Unique microbes in samples}

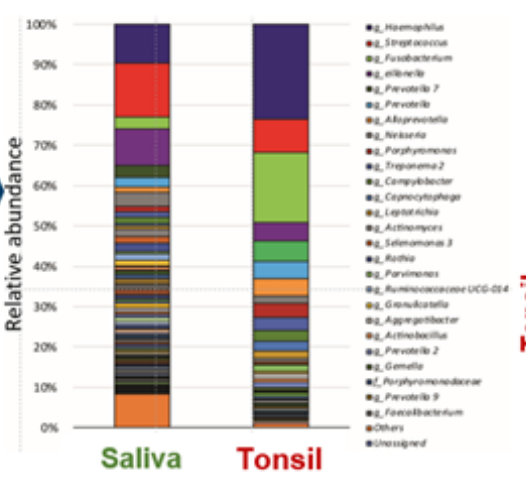

Correlations between samples

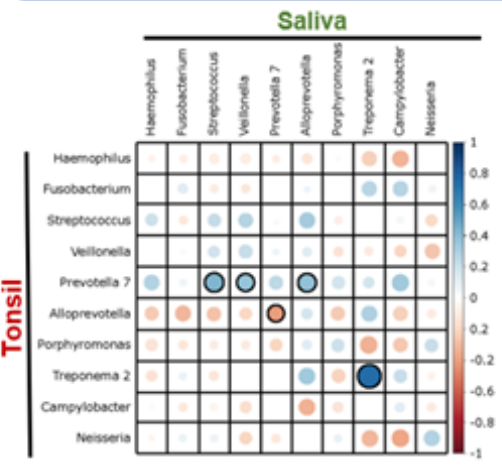

Figure 6

Overview of the experimental framework.

\section{Supplementary Files}

This is a list of supplementary files associated with this preprint. Click to download.

- Additionalfile1.xlsx

- Additionalfile2.xlsx

- Additionalfile1.xlsx

- Additionalfile2.xlsx 